\title{
Congenital Epidermal Cyst of the Umbilicus: A Case Report*
}

\author{
Noriko Aramaki-Hattori ${ }^{1 \#}$, Takara Tanaka ${ }^{2}$, Kiyoshi Mukai ${ }^{3}$, Kazuo Kishi $^{1}$ \\ ${ }^{1}$ Department of Plastic and Reconstructive Surgery, Keio University Hospital, Tokyo, Japan; ${ }^{2}$ Department of Plastic and Reconstruc- \\ tive Surgery, Saiseikai Central Hospital, Minato-ku, Japan; ${ }^{3}$ Department of Pathology, Saiseikai Central Hospital, Minato-ku, Japan. \\ Email: \#nonken@2001.jukuin.keio.ac.jp
}

Received July 29 ${ }^{\text {th }}, 2013$; revised August $28^{\text {th }}, 2013$; accepted September $5^{\text {th }}, 2013$

Copyright (c) 2013 Noriko Aramaki-Hattori et al. This is an open access article distributed under the Creative Commons Attribution License, which permits unrestricted use, distribution, and reproduction in any medium, provided the original work is properly cited.

\begin{abstract}
Epidermal cysts are one of the most common benign skin tumors, although they are rarely found in the umbilicus of a child. Herein we report the case of a 13-year-old girl with a congenital umbilical mass. This mass had been misdiagnosed as a lipoma at birth. Awareness of this lesion can avoid its misdiagnosis and inappropriate treatment.
\end{abstract}

Keywords: Congenital; Epidermal Cyst; Umbilicus

\section{Case Report}

A 13-year-old girl presented with a red, swollen mass in her umbilicus (Figure 1). This mass had been present since birth, and was diagnosed as a lipoma by her pediatrician. She was asymptomatic except for occasional slight pain at the lesion. There was no history of tumor rupture. Magnetic resonance imaging revealed a subcutaneous mass which did not penetrate into the abdominal cavity or contact any internal organs (Figure 2). The mass ruptured 1 day prior to surgery, and on the day of the operation the tumor had developed a large hole and was crusted over. Total excision was performed under local anesthesia. Exploration during the operation showed that the mass did not have any duct connecting it to the abdominal cavity. The patient's postoperative course was uneventful. The sutures were removed after 10 days, and the patient had a good cosmetic result (Figure 3). Histological assessment revealed an epidermal cyst; there was no malignancy (Figure 4).

\section{Discussion}

Epidermal cysts are common, benign skin tumors usually involve the scalp, face, neck, back, and trunk [1]. A cyst may rupture, and extrusion of its keratin into the dermis may incite a foreign body reaction [2]. Epidermal cysts are usually asymptomatic unless they get infected or

\footnotetext{
*Conflict of interest: None declared. Funding: None. Ethical approval: Not required.

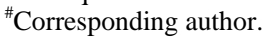

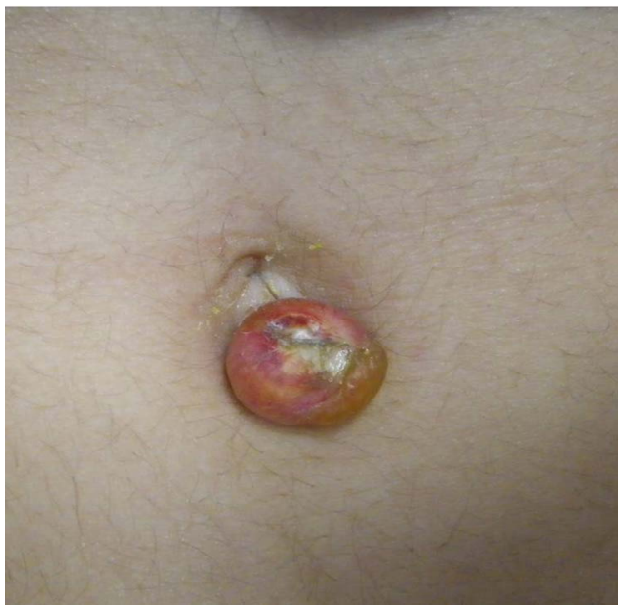

Figure 1. Pre-operative photograph of the umbilical mass.

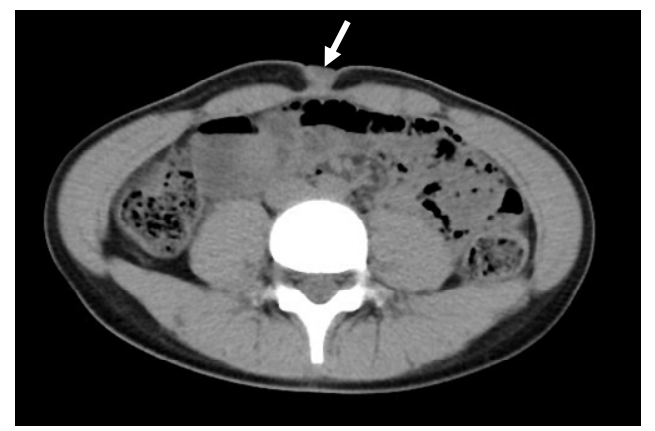

Figure 2. Magnetic resonance imaging of the abdomen showing a subcutaneous mass, not communicating with the abdominal cavity (see mass adjacent to white arrow). 


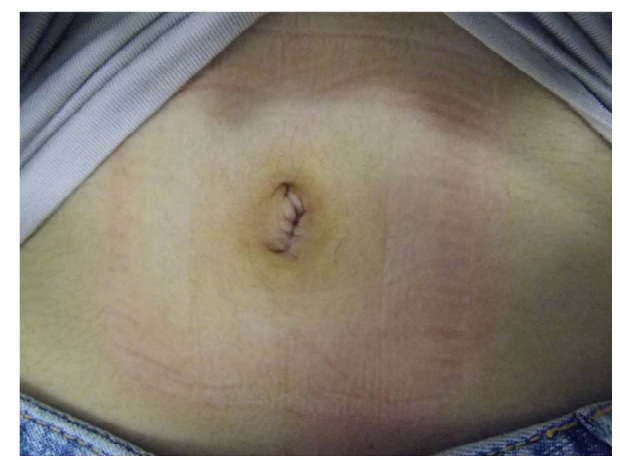

Figure 3. Post-operative photograph of the umbilicus.

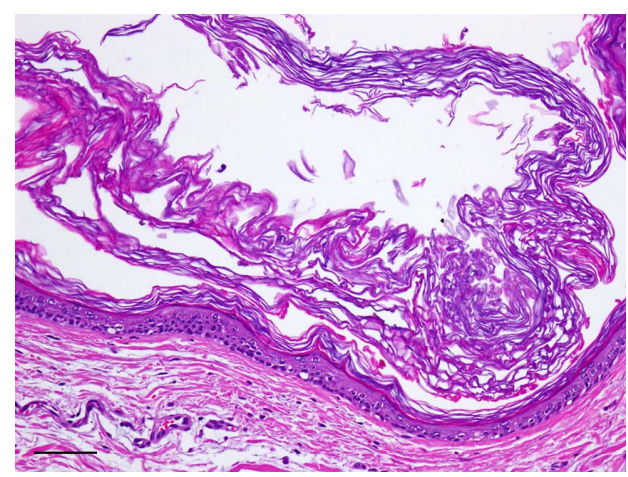

Figure 4. Hematoxylin and eosin staining reveals an epidermal cyst (Bar: $100 \mu \mathrm{m})$.

enlarge sufficiently to damage adjacent anatomic structures [3]. In this patient's case, the tumor was stable for 13 years, rupturing 1 day prior to surgery.

Other types of common, benign skin tumors as a differential diagnosis are dermoid cysts, lipomas, and calcifying epitheliomas (pilomatrixomas). Dermoid cysts, one of the common congenital skin tumors, differ from epidermal cysts in that they have skin structures such as hair or glands inside the tumor. Although there are a few reports of epidermal inclusion cysts at the umbilicus following abdominoplasty [4,5], we were not able to locate any report of congenital epidermal cysts in this location.

The differential diagnosis of congenital lesions of the umbilicus includes umbilical hernia, umbilical cord hernia, umbilical polyp, and urachal remnant. By the time of birth, the umbilical ring has become entirely closed by the developing abdominal wall except for the space occupied by the cord, which contains the umbilical vein, paired umbilical arteries, and the fibrous remnants of the urachus (allantois), and omphalomesenteric duct (yolk sac) [6]. Final closure of the umbilical ring does not occur until separation of the cord at birth [7]. After ligation of the cord, the vessels thrombose, and the cord dries and sloughs, leaving a granulating surface that heals by cicatrization becomes covered by epithelium [6]. The mechanism of formation for epidermal cysts is still unclear, but in this case we suspect that the involution of an epidermal component into the dermis at birth may have caused a congenital epidermal cyst.

\section{REFERENCES}

[1] S. G. Kang, C. H. Kim, H. K. Cho, M. Y. Park, et al., "Two Cases of Giant Epidermal Cyst Occurring in the Neck,” Annals of Dermatology, Vol. 23, Suppl. 1, 2011, pp. 135-138. http://dx.doi.org/10.5021/ad.2011.23.S1.S135

[2] K. Matsumono, H. Okabe, M. Ishizawa and M. Egawa, "Intermetatarsophalangeal Bursitis Induced by a Plantar Epidermal Cyst,” Clinical Orthopaedics and Related Research, Vol. 385, 2001, pp. 151-156. http://dx.doi.org/10.1097/00003086-200104000-00023

[3] Y. Basterzi, A. Sari and S. Ayhan, "Giant Epidermoid Cyst on the Forefoot,” Dermatologic Surgery, Vol. 28, No. 7, 2002, pp. 639-640. http://dx.doi.org/10.1046/j.1524-4725.2002.01314.x

[4] A. A. Andreadis, C. M. Samson, S. Szomstein and I. M. Newman, "Epidermal Inclusion Cyst of the Umbilicus Following Abdominoplasty,” Plastic Surgical Nursing, Vol. 27, No. 4, 2007, pp. 202-205.

[5] C. C. Camenisch and P. Heden, "Umbilical Epithetlial Cyst in Secondary Abdominoplasty: Case Report,” Aesthetic Plastic Surgery, Vol. 36, No. 1, 2012, pp. 83-87.

[6] J. A. O’Neil Jr., J. L. Grosfeld and E. W. Fonkalsrud, "Principles of Pediatric Surgery," 2nd Edition, Mosby, Missouri, 2004.

[7] S. J. Mathes, "Mathes Plastic Surgery," 2nd Edition, Vol. 6, Saunders, Philadelphia, 2006. 